\title{
Usefulness of Botulinum Toxin Type-A in the Treatment of Chronic Sixth Nerve Palsy
}

\author{
Lelio Sabetti ${ }^{*}$, Immacolata Greco, Domenico Di Lodovico, Antonio Berarducci
}

Eye Clinic, San Salvatore Hospital, University of L'Aquila, L'Aquila, Italy.

Email: *studiosabetti@yahoo.it

Received November $7^{\text {th }}, 2011$; revised December $16^{\text {th }}, 2011$; accepted January $28^{\text {th }}, 2012$

\begin{abstract}
Purpose: The purpose of the study was to evaluate the efficacy of botulinum toxin type A (BTX-A) injection in patients with acquired VI nerve palsy. Methods: Twenty-eight patients (16 F and $12 \mathrm{M}$ ), mean age $36.4+/-17.8 \mathrm{DS}$; range 10 69 years with acquired VI nerve palsy, have been treated with BTX-A injection into the ipsilateral medial rectus, at least 6 months after onset. At 6 months follow up, a paresis and a paralysis, were respectively diagnosed if the affected eye could be actively abducted or not, across the mid-line; a simple horizontal rectus muscle procedure was performed for the paresis whilst for the paralysis, both a horizontal and vertical muscle transportation procedure were required. Results: A gradual physiological recovery of the lateral rectus muscle was observed in $9(32 \%)$ patients (GROUP I) and surgical treatment was therefore unnecessary; the remaining 19 cases (68\%), of which 5 paresis (GROUP II) and 14 paralysis (GROUP III) underwent surgery and within 1 year were all restored to orthotropia. Conclusion: Botulinum toxin type-A (BTX-A) injection is an invaluable tool in the differential diagnosis between paresis versus paralysis of the VIth nerve, allowing the correct choice of surgical procedure.
\end{abstract}

Keywords: Chronic Acquired VI Nerve Palsy; Botulinum Toxin Type-A

\section{Introduction}

Acquired sixth nerve palsy is usually a consequence of vascular or neurological diseases, diabetes, head trauma $[1,2]$ or cerebral tumors [3]. Diagnosis and management of this neurogenic ocular palsy are often difficult largely due to the fact that the ophthalmologist has insufficient and/or inaccurate clinical history of the patients. It is generally necessary, prior to surgical treatment, to wait at least for 6-8 months, in order for the paralysis to be considered stable. A spontaneous recovery of this paralysis is rare [4]. It is important to note, however, that there is a frequent occurrence of the contraction of the joint antagonist muscle which may mask the functional recovery of the latter. Patients thus affected manifest large angle isotropia. Adults experience double vision and are forced to tilt the head to neutralize diplopia or to use prismatic lenses. If diplopia is not adequately controlled it may require surgical treatment, the functional result of which is often however unpredictable. Botulinum toxin type-A (BTX-A) injected in the hyperfunctional muscles causes a flaccid paralysis by preventing the release of acetylcholine at the neuromuscular junction and thus results in a gradual physiological recovery of the hypofunctional

\footnotetext{
${ }^{*}$ Corresponding author.
}

muscles [5]. BTX-A injection can be used either during the waiting period for the spontaneous recovery or for surgery; in cases where VI nerve palsy is resolved, BTX-A help to prevent diplopia, whereas in the case of unresolved palsy, the toxin is an invaluable tool in the differential diagnosis between paresis versus palsy, which in turn allows the correct choice of surgical procedure.

\section{Materials and Methods}

Between April 2001 and February 2007, twenty-eight consecutive patients were referred to our clinic with acquired sixth nerve palsy. The 16 women and the 12 men were 10 to 69 years of age (mean $36.4+/-17.8 \mathrm{SD}$ ). All patients underwent a complete preoperative ophthalmological and orthoptic clinical protocol, including ocular refraction with and withuot cycloplegia, Goldmann's tonometry, biomicroscopy of the anterior segment and funduscopic examination. The orthoptic examination evaluated the ocular motility in the nine diagnostic gaze positions; strabismus was measured with the prism and cover test for near and distance fixation and presence of anomalous head positions was identified, Worth and micro-Worth 4-dot test, Lang test (I-II), Titmus, red filter ladder test, Hess-Lancaster screen test and passive and 
active abduction test were also performed. Mean preinjection angle deviation was $22^{\circ}$ ET (+/- $\left.0.64 \mathrm{SD}\right)$ near and $24.9^{\circ}$ ET (+/- $\left.0.74 \mathrm{SD}\right)$ distance. Inclusion criteria were sixth nerve palsy more than 6 months after onset and an anomalous position of the head. The patients were divided into 3 class according the time of onset of the palsy: 9 patients (CLASS I) with more than 24 months after onset; 11 patients (CLASS II) between 12 and 24 months after onset; 8 patients (CLASS III) between 6 and 12 months after onset. Exclusion criteria were: previous surgical treatments, congenital abnormalities of extraocular muscles and cases where there had been administration of aminoglycosides or other drug causing interference with the neuromuscular system, in the last 6 months. Appropriate informed consent was obtained in all cases. Each patient underwent a single injection of BTX-A (20 IU, Botox, Dysport ${ }^{\circledR}$, Ipsen) under local anaesthesia directly in the ipsilateral medial rectus muscle.

\section{Results}

Follow ups were performed at 1 and 6 months intervals. At 6 months the differential diagnosis between a paresis and paralysis was respectively defined if the affected eye could or could not be actively abducted or not across the midline. Patients with paresis underwent a single horizontal rectus muscle procedure, whilst patients with paralysis further underwent horizontal and vertical muscle transposition procedure $[6,7]$. The patients were divided into 3 groups. Nine of them (32\%) (group I) manifested a gradual physiological recovery of the lateral rectus muscle at 1 month follow up (mean $4.57^{\circ} \mathrm{ET}+/-0.90 \mathrm{SD}$ near and $3.42^{\circ} \mathrm{ET}+/-0.51 \mathrm{SD}$ distance). This group was composed of 4 patients with head trauma, 3 patients with vascular diseases, 1 patient with diabetes and 1 patient with an idiopathic case; six of them belonged to CLASS I, one of them belonged to CLASS II and two of them belonged to CLASS III. These patients complained of diplopia within only two months of the BTX-A injection but this condition could be neutralized with prismatic lenses. Consequently these patients did not require a surgical procedure. In contrast, at 1 and 6 months follow up, five patients (18\%) (group II) manifested paresis with an active abduction across the mid-line, at 1 month (mean $14.28^{\circ} \mathrm{ET}+/-0.90 \mathrm{SD}$ near and $18.28^{\circ} \mathrm{ET}+/-0.90 \mathrm{SD}$ distance). This group was composed of two patients with vascular diseases, 1 patient with head trauma, 1 patient with diabetes and 1 patient with cerebral tumor; two of them belonged to CLASS I, two of them belonged to CLASS II and one of them belonged to CLASS III. These patients underwent horizontal procedure of muscle recession and/or resection. Finally, 14 cases (50\%) (group III) manifested paralysis without an active abduction across the mid-line, at 1 month (mean $21.34^{\circ}$ ET +/-
1.06 SD near and $22.28^{\circ} \mathrm{ET}+/-0.74 \mathrm{SD}$ distance) and at 6 months follow up (mean $23.05^{\circ} \mathrm{ET}+/-0.91 \mathrm{SD}$ near and $24.48^{\circ} \mathrm{ET}+/-0.86 \mathrm{SD}$ distance) consequently all of them underwent a horizontal as well as a vertical muscle transposition procedure. This group was composed of 6 patients with head trauma, 3 patients with vascular diseases, 3 patients with cerebral tumors, 1 patient with diabetes and 1 patient with an idiopathic case; one of them belonged to CLASS III. Within a year of surgery all patients were either restored to orthotropia or remained with a small-angle residual isotropy in primary position, easily corrected with lenses.

\section{Discussion}

The aim of surgery in paralytic strabismus is to restore the best equilibrium between muscle forces unbalanced by ocular palsy. Moreover it is necessary to eliminate the eventual presence of diplopia and/or of anomalous head posture. In the VI nerve palsy the choice of the surgical strategy could depend on the extent of recovery induced by BTX-A injection, which thus becomes an invaluable tool in surgical evaluation [8]. During the first 3 months after the onset of palsy, it is possible to have a spontaneous recovery, but in the following period there is often the occurrence of contraction of the joint antagonist muscle. Elasticity of the deficient muscle is gradually reduced due to a constant elongation resulting in a gradual loss of tonus, hyper-extensibility and progressive degeneration of muscolar fibres [9]. According to available literature, the degeneration becomes irreversible after one or two years of the reduction of active muscle tension, and the high-resolution MRI can directly demonstrate the degeneration of muscolar fibres [10]. However, as demonstrated in this study, a full recovery $(24 \%)$ or a partial recovery of the lateral rectus function $(20 \%)$ are possible even in long term VI nerve palsy [11]. BTX-A injection in acquired sixth nerve palsy, avoids a hyper-contraction of the ipsilateral medial rectus muscle [12], promotes the recovery of the lateral rectus function and eliminates the need of surgical intervention in patients who can benefit from restored orthotropia by a sole injection. Patients who manifest a persistent large angle isotropy with diplopia or anomalous head position, allow for a differential diagnosis between a paresis vs paralysis; as previously stated, this comparison becomes instrumental in establishing the need of surgery. If the affected eye cannot be abducted beyond the mid-line, the transposition procedure will be required; if eye abduction is possible the recession and resection procedure should be sufficient. Botulinum toxin treatment was originally developed 25 years ago by Ala B. Scott to produce reversible weakening of extraocular eye muscle (chemodenervation) $[2,13]$ in the treatment of strabismus. Today it is 
an important aid in the preoperative evaluation of possible postoperative diplopia on patients in which this cannot be done by means of prism or traction test; in acute paretic loss of ocular muscle function; when surgical treatment of the ocular muscles is not yet possible but the patient is obviously affected by diplopia or a forced posture of the head [2], in situations where strabismus surgery is not suitable (as in elderly patients unfit for general anaesthesia; when the clinical condition is evolving or unstable, or in case of unsuccessful surgery) [13], in acute Graves' disease, and especially into VI cranial nerve paresis [2], or in association with the surgery [14]. Depending on the surgical approach in VI nerve palsies, Botulinum toxin may be injected in the medial rectus muscle before muscle transposition surgery to loosen contracture [2] or, on the basis of our results, BTX-A injection can be considered an invaluable tool in the management and diagnosis of VI nerve palsy, and it allows differential diagnosis between paresis vs paralysis, which is the necessary condition to plan the correct surgical treatment and to obtain a successful functional outcome. In other strabismus, as congenital esotropia or horizontal strabismus in adults, Botulinum toxin is definitely an inferior surgical treatment of the ocular muscles [2]. Moreover the use of botulinum toxin type A is efficient in the identification of adult patients with constant strabismus who are at high risk o developing intractable diplopia after surgery [15]. The majority of studies identify the Botulinum toxin A injection into extra-ocular muscles the ideal therapy for the treatment of sixth cranial nerve palsy [1], on $36.8 \%$ of the patients with a final ocular alignment within 10 prism dioptres of orthotropia and achieved fusion and primary gaze position. There was no correlation among the number of injection for patient (the mean number of injection is 1 to 5 ), the size of strabismus, the grade of lateral rectus muscle function, the age, the gender or the time paresis it occurred [1]. In our study all patients had to undergo a single injection and only in $32 \%$ of cases ( 9 patients) a gradual physiological recovery of the lateral rectus muscle was obtained at 6 months like if the surgical treatment could be avoided and nobody of them was candidate for a second injection; patients under-corrected at six months from the onset were been treated with surgery. The most interesting result was to identify in GROUP 1 six patients who had been suffering with palsy for a period in excess of 24 months before the injection (CLASS I); although it is known and documented that VI nerve deficit of traumatic origin tends to normalize without any treatment or after botulinum toxin injection $[7,16,17]$ this has never been recorded for cases where patients have been subject to palsy for such a long time. Moreover it is also possible to combine the injection with surgery [14] to preserve the function of the medial rectus muscle, in order to avoid surgery on more than two rectus muscles to prevent the risk of anterior segment ischemia (which can occur when there is surgery of multiple muscles) and to expand the field of binocular single vision as much as possible. In our trials, Botulinum toxin type-A (BTX-A) was directly injected in the hyperfunctional muscle without electromyographic assistance, in order to reduce the incidence of blepharoptosis [18]; a study of 2003 proposed that subtenon injection of the medial rectus muscle for the treatment of acute tramatic sixth nerve palsy [19]. The results on the patients treated with subtenonian injection of botulinum toxin without electromyography (EMG) guidance was comparable to that obtained using EMGguided intramuscolar injection of botulinum toxin [19, 20], the reason is in fact that the substance injected in the muscle has the tendency to diffuse into the surrounding tissues. Finally BTX injection has been used for 11 years by 292 ophthalmologists in 8854 patients, from the age of three months to 90 years, in a variety of eye muscle and eyelid disorders [21]. According to international literature, no systemic toxin reaction has occurred, local complications are few and visual loss has not occurred in any case [21]; ptosis and acquired vertical deviation caused by diffusion of the substance into the surrounding tissues are the commonest complications encountered. These are minor and reversible damages induced by the traumatic action of the needle and/or the substance into the tissues (edema and minor subconjunctival hemorrhage), as further proof that the repeated use of BTX-A could be considered safe [13].

\section{REFERENCES}

[1] H. L. Hung, L. Y. Kao and M. H. Sun, "Botulinum Toxin Treatment for Acute Traumatic Complete Sixth Nerve Palsy," Eye, Vol. 19, No. 3, 2005, pp. 337-341. doi:10.1038/sj.eye. 6701460

[2] T. Krzizok, "Botulinum Toxin Injections for the Treatment of Strabismus. Which Indications Are Still Useful Today?" Ophthalmology, Vol. 104, No. 9, 2007, pp. 759-762.

[3] S. Gujar, J. Trobe and D. Gandhi, "Spontaneous Resolution of a Meckel's Cave Arachnoid Cyst Causing Sixth Cranial Nerve Palsy," Journal of Neuro-Ophthalmol, Vol. 28, No. 3, 2008, pp. 186-191. doi:10.1097/WNO.0b013e318183be6c

[4] J. M. Holmes, D. A. Leske and S. P. Christiansen, "Initial Treatment Outcomes in Chronic Sixth Nerve Palsy," Journal of AAPOS, Vol. 5, No. 6, 2001, pp. 370-376. doi:10.1067/mpa.2001.120176

[5] L. Kowal, E. Wong and C. Yahalom, "Botulinum Toxin in the Treatment of Strabismus. A Review of Its Use and Effects," Disability and Rehabilitation, Vol. 29, No. 23, 2007, pp. 1823-1831. doi:10.1080/09638280701568189

[6] A. B. Scott, "Botulinum Toxin Injection into Extraocular Muscles as an Alternative to Strabismus Surgery," Oph- 
thalmology, Vol. 10, 1980, pp. 1040-1049.

[7] P. Riordan-Eva and J. P. Lee, "Management of VIth Nerve Palsy-Avoiding Unnecessary Surgery," Eye, Vol. 4, 1992, pp. 386-390. doi:10.1038/eye.1992.79

[8] J. S. Elston and J. P. Lee "Paralytic Strabismus: The Role of Botulinum Toxin," British Journal of Ophthalmology, Vol. 69, No. 12, 1985, pp. 891-896. doi:10.1136/bjo.69.12.891

[9] G. Kommerell, D. Olivier and H. Theopold, "Adaptive Programming of Phasic and Tonic Components in Saccadic Eye Movements. Investigations of Patients with Abducens Palsy," Investigative Ophthalmology \& Visual Science, Vol. 15, No. 8, 1976, pp. 657-660.

[10] J. L. Demer, M. C. Ortube, E. C. Engle and N. Thacker, "High-Resolution Magnetic Resonance Imaging Demonstrates Abnormalities of Motor Nerves and Extraocular Muscles in Patients with Neuropathic Strabismus," Journal of AAPOS, Vol. 10, No. 2, 2006, pp. 135-142. doi:10.1016/j.jaapos.2005.12.006

[11] J. Acheson, C. Bentley, J. Shallo-Hoffmann and M. Gresty, "Dissociated Effects of Botulinum Toxin Chemodenervation on Ocular Deviation and Saccade Dynamics in Chronic Lateral Rectus Palsy," British Journal of Ophthalmology, Vol.1, No. 82, 1998, pp. 67-71. doi:10.1136/bjo.82.1.67

[12] A. G. Altintas, H. B. Arifoglu, D. Dal and S. Simsek, "Are Most Sixth Nerve Palsies Really Paralytic?" Journal of Pediatric Ophthalmology Strabismus, Vol. 48, No. 3, 2011, pp. 187-191. doi:10.3928/01913913-20100618-01

[13] L. Kowal, E. Wong and C. Yahalom, "Botulinum Toxin in the Treatment of Strabismus. A Review of Its Use and Effects," Disability and Rehabilitation, Vol. 29, No. 23, 2007, pp. 1823-1831. doi:10.1080/09638280701568189

[14] R. Fitzsimons, J. P. Lee and J. Elston, "Treatment of Sixth Nerve Palsy in Adults with Combined Botulinum
Toxin Chemodenervation and Surgery," Ophthalmology, Vol. 95, No. 11, 1988, pp. 1535-1542.

[15] J. Khan, I. Kumar and I. B. Marsh, "Botulinum Toxin Injection for Postoperative Diplopia Testing in Adult Strabismus," Journal of American Association for Pediatric Ophthalmology and Strabismus, Vol. 12, No. 1, 2008, pp.46-48.

[16] J. O. Lee, "Modern Management of Sixth Nerve Palsy," Australian and New Zealand Journal of Ophthalmology, Vol. 20, No. 1, 1992, pp. 41-46.

[17] J. Lee, S. Harris, K. Cooper, C. MacEwen and S. Jones, "Results of a Prospective Randomized Trial of Botulinum Toxin Therapy in Acute Unilateral Sixth Nerve Palsy," Journal of Pediatric Ophthalmology Strabismus, Vol. 31, No. 5, 1994, pp. 283-286.

[18] M. S. Sanjari, K. G. Falavarjani, M. B. Kashkouli, G. H. Aghai, M. Nojomi and H. Rostami, "Botulinum Toxin Injection with and without Electromyographic Assistance for Treatment of Abducens Nerve Palsy: A Pilot Study," Journal of AAPOS, Vol. 12, No. 3, 2008, pp. 259-262. doi:10.1016/j.jaapos.2007.11.006

[19] L. Y. Kao and A. N. Chao, "Subtenon Injection of Botulinum Toxin for Treatment of Traumatic Sixth Nerve Palsy," Journal of Pediatric Ophthalmology Strabismus, Vol. 40, No. 1, 2003, pp. 27-30.

[20] E. C. Benabent, P. García Hermosa, M. T. Arazola and J. L. Alió y Sanz, "Botulinum Toxin Injection without Electromyographic Assistance," Journal of Pediatric Ophthalmology Strabismus, Vol. 39, No. 4, 2002, pp. 231-234.

[21] No Authors Listed, "Botulinum Toxin Therapy of Eye Muscle Disorders. Safety and Effectiveness. American Academy of Ophthalmology," Ophthalmology, 1989, pp. 37-41. 